\title{
Development of a Simple Tool for Identifying Alcohol Use Disorder in Female Korean Drinkers from Previous Questionnaires
}

\author{
Yu Ri Seo, Jong Sung Kim*, Sung Soo Kim, Seok Joon Yoon, Won Yoon Suh, Kwangmi Youn \\ Department of Family Medicine, Research Institute for Medical Sciences, Chungnam National University School of Medicine, Daejeon, Korea
}

\begin{abstract}
Background: This study aimed to develop a simple tool for identifying alcohol use disorders in female Korean drinkers from previous questionnaires.

Methods: This research was conducted on 400 women who consumed at least one alcoholic drink during the past month and visited the health promotion center at Chungnam National University Hospital between June 2013 to May 2014. Drinking habits and alcohol use disorders were assessed by structured interviews using the Diagnostic and Statistical Manual of Mental Disorders, 5th Edition diagnostic criteria. The subjects were also asked to answer the Alcohol Use Disorders Identification Test (AUDIT), AUDIT-Consumption, CAGE (Cut down, Annoyed, Guilty, Eye-opener), TWEAK (Tolerance, Worried, Eye-opener, Amnesia, Kut down), TACE (Tolerance, Annoyed, Cut down, Eye-opener), and NET (Normal drinker, Eye-opener, Tolerance) questionnaires. The area under receiver operating characteristic (AUROC) of each question of the questionnaires on alcohol use disorders was assessed. After combining two questions with the largest AUROC, it was compared to other previous questionnaires.

Results: Among the 400 subjects, 58 (14.5\%) were identified as having an alcohol use disorder. Two questions with the largest AUROC were question no. 7 in AUDIT, "How often during the last year have you had a feeling of guilt or remorse after drinking?" and question no. 5 in AUDIT, "How often during the past year have you failed to do what was normally expected from you because of drinking?" with an AUROC (95\% confidence interval [CI]) of 0.886 (0.850-0.915) and $0.862(0.824-0.894)$, respectively. The AUROC (95\% CI) of the combination of the two questions was 0.958 (0.934-0.976) with no significant difference as compared to the existing AUDIT with the largest AUROC. Conclusion: The above results suggest that the simple tool consisting of questions no. 5 and no. 7 in AUDIT is useful in identifying alcohol use disorders in Korean female drinkers.
\end{abstract}

Keywords: Alcohol Drinking; Female; Surveys and Questionnaires 


\section{INTRODUCTION}

In recent decades, alcohol consumption has increased in Korean women due to several factors, including changes in social perception and norms associated with drinking, improved education levels, and increased participation in the workforce. According to alcohol consumption/ingestion-related statistics gathered by the Korea Food \& Drug Administration in 2012, ${ }^{1)}$ the percentage of women who were at high risk of drinking more than 5 glasses of soju in one drinking session once a week increased from $14.3 \%$ in 2011 to $18.1 \%$ in 2012 , and that of those who drank more often than twice a week increased from $7.7 \%$ in 2011 to $11.1 \%$ in 2012. According to the 2012 National Health and Nutrition Examination Survey carried out by the Department of Health and Human Services, ${ }^{2)}$ the percentage of highrisk drinking in females was $6 \%$, the weekly percentage of excessive drinking of more than 5 glasses in one drinking session more often than once a week for the past year was $14.6 \%$, and the percentage of problem drinking was $12.9 \%$.

Several studies of problem drinking report that a systematic approach to alcohol abuse disorders can reduce alcohol abuse in patients. ${ }^{3-5)}$ Since primary care doctors often encounter patients who have problems with alcohol, they should be able to use effective screening methods to discover problem drinkers.

Screening of problem drinking often involves use of the Cut down, Annoyed, Guilty, Eye-opener (CAGE) and Alcohol Use Disorders Identification Test (AUDIT). The AUDIT is known to be effective in screening all at-risk drinking, problem drinking, and alcohol use disorders, ${ }^{6)}$ but it is somewhat difficult to carry out in the primary care setting due to its relatively long administration time. CAGE questionnaires consist of 4 questions, taking less time to administer, which is appropriate for outpatients in primary care. ${ }^{7)}$ However, there are studies prove that Tolerance, Annoyed, Cut down, Eye-opener (TACE) or Tolerance, Worried, Eye-openers, Amnesia, Kut down(TWEAK) is more useful than CAGE for obstetric patients. ${ }^{8,9)}$ TACE and TWEAK are suggested as the most effective tools to detect pregnant problem drinkers; ${ }^{10)}$ they are easy to score and apply and are more sensitive at assessing at-risk drinking in pregnant women. ${ }^{11)}$

AUDIT is a screening tool that is often used in primary care, but is problematic as it is complex and takes much time to screen patients. ${ }^{12,13)}$ Consequently, interest in developing a simpler, shorter and more effective screening tool is increasing. Accordingly, there were several attempts to make a questionnaire that consisted of 1 or 2 questions. ${ }^{14,15)}$ However, they lost currency as further research did not progress in the clinical setting.

Both globally and in Korea the prevalence of women's alcohol use disorders is increasing, and drinking seems to have a greater impact on households and communities when it occurs in women, making it especially important to screen and manage female drinkers. However, most studies are conducted on male drinkers or pregnant women who drink, leading to large numbers of undetected female alcohol users. Thus, this study aimed to find a useful and easy tool for screening female problem drinkers through the recombination of existing questionnaires.

\section{METHODS}

\section{Subjects}

This study was conducted on 2,840 women who visited the health promotion center at Chungnam National University Hospital between June 2013 and May 2014. The participants were 400 women who had drunk alcohol within the past month and had agreed to participate in the study.

\section{Methods}

\section{1) Diagnostic interview}

Through diagnostic interviews with a family doctor, the status of at-risk drinking and alcohol use disorders was assessed. First, to assess the status of heavy drinking and excessive drinking, subjects' drinking quantity per time, the number of drinking sessions per week, and the largest drinking quantity were investigated. At this time, according to the criteria of the National Institute on Alcohol Abuse and Alcoholism (NIAAA), ${ }^{16)}$ pure alcohol of $14 \mathrm{~g}$ was calculated as a standard drinking unit, and $90 \mathrm{~mL}$ of $20 \%$ soju (1/4 bottle), a small bottle of beer, 1 bowl of rice wine or 1 glass of wine were converted to the standard drink unit of $14 \mathrm{~g}$.

According to the NIAAA criteria for female drinking, a largest drinking quantity exceeding 3 standard drink units at one time was defined as binge drinking, and drinking over 7 standard drink units per week was defined as heavy drinking. A case that came under heavy drinking or binge drinking was defined as at-risk drinking. Presence of alcohol use disorder was assessed by using a method of 'structured interviews' according to the diagnostic criteria of the Diagnostic and Statistical Manual of Mental Disorders, 5th edition (DSM-5). ${ }^{17)}$

\section{2) Survey}

The subjects were asked to respond to 10 questions in AUDIT, 4 in CAGE, 5 in TWEAK, 4 in TACE, and 3 in Normal drinker, Eye-opener, Tolerance (NET). In addition, socio-demographic characteristics including age, educational background, religious status, marriage status, smoking status, and age of first drinking were surveyed.

\section{3) Research tools}

(1) Alcohol Use Disorders Identification Test

AUDIT is a 10-question questionnaire developed by the World Health Organization in 1989, with a value of 4 points for each 
question for a total of 40 points. ${ }^{6}$

"How often do you have a drink containing alcohol?" (question no. 1), "How many drinks containing alcohol do you have on a typical day when you are drinking?" (no. 2), "How often do you have six or more drinks on one occasion?" (no. 3), "How often during the last year have you found that you were not able to stop drinking once you had started?" (no. 4), "How often during the last year have you failed to do what was normally expected from you because of drinking?" (no. 5), "How often during the last year have you needed a first drink in the morning to get yourself going after a heavy drinking session?" (no. 6), "How often during the last year have you had a feeling of guilt or remorse after drinking?" (no. 7), "How often during the last year have you been unable to remember what happened the night before because of your drinking?" (no. 8), "Have you or someone else been injured because of your drinking?" (no. 9), and "Has a relative, friend, doctor, or other health care worker been concerned about your drinking or suggested you cut down?" (no. 10).

This study used AUDIT-Korean revised, which modified the quantity of the 'standard drink unit' in the questionnaire according to the guidelines proposed by the NIAAA in $2007 .{ }^{18,19)}$

(2) Alcohol Use Disorders Identification Test-Consumption Alcohol Use Disorders Identification Test-Consumption (AUDIT-C) ${ }^{20,21)}$ is calculated by adding up the scores of responses to questions 1 through 3, asking "the number of times of drinking," "drinking quantity in ordinary times," and "frequency of binge drinking," of the 10 questions of the AUDIT, and the maximum score is 12 points.

(3) Cut down, Annoyed, Guilty, Eye-opener

CAGE is a questionnaire developed in 1970, consisting of 4 questions, "Have you ever felt the need to cut down on your drinking?" (cut-down), "Have you ever felt annoyed by criticism of your drinking?" (annoyed), "Have you ever felt guilty about your drinking?" (guilty), and "Have you ever felt the need to drink a morning eye-opener?” (eye-opener).

(4) Tolerance, Annoyed, Cut down, Eye-opener

TACE consists of 4 questions, designed by Sokol et al. ${ }^{8)}$ to assess at-risk drinking in pregnant women. It is a questionnaire that substituted the question about guilt in CAGE with a question about tolerance: "How many drinks does it take to feel the first effect?" This consists of 4 questions about tolerance, annoyance, cut down and eye-opener and judges over 2 points to be at-risk drinking.

(5) Tolerance, Worried, Eye-opener, Amnesia, Kut down TWEAK consists of 5 questions, developed by Russell et al. ${ }^{11)}$ to screen at-risk drinking during pregnancy. It consists of 5 ques- tions: "How many drinks does it take to feel the first effect?" (tolerance), "Have close friends worried or complained about your drinking in the past year?" (worried), "Do you sometimes take a drink in the morning when you first get up?" (eye-openers), "Has a friend or family member ever told you about things you said or did while you were drinking that you could not remember?" (amnesia), and "Do you sometimes feel the need to cut down on tour drinking?" (cut-down), and it is considered very likely to be problem drinking if one has 3 points or higher.

\section{(6) Normal drinker, Eye-opener, Tolerance}

NET is a questionnaire developed by Bottoms et al. ${ }^{22)}$ It consists of 3 questions which measure heavy drinking in women in their childbearing years. This questionnaire asks whether one thinks oneself to be a normal drinker, whether one has ever had a morning drink (eye-opener), and how many glasses one needs to feel intoxicated (tolerance: positive if over 3 glasses).

\section{Data Processing}

Area under receiver operating characteristic (AUROC) curve was calculated by the receiver operating characteristic (ROC) curves on the screening of alcohol use disorders for each question of the screening tools. The difference between areas for each question were compared using the comparison of independent ROC curves. For statistical analyses, IBM SPSS Win ver. 20.0 (IBM Co., Armonk, NY, USA) and Medcalc Statistical Software ver. 12.7 (MedC Software, Mariakerke, Belgium) were used, and the level of statistical significance was set to P-value below 0.05 .

\section{RESULTS}

\section{General Characteristics and Alcohol-Related Characteristics of Research Subjects}

The mean age of subjects \pm standard deviation (SD) was $42.2 \pm$ 12.8 years old, 234 of them had a religion (58.5\%), 330 were living with their guardians (82.5\%), 7 were smokers (1.8\%), 9 were elementary school graduates $(2.3 \%), 38$ were middle school graduates (9.5\%), 122 were high school graduates (30.5\%), and 211 were college graduates $(52.8 \%)$. The mean age of starting drinking was $22.5 \pm 6.3$ years old; the mean frequency of drinking per week was $0.5 \pm 0.7$ times; the mean quantity per time was $1.6 \pm 1.6$ drinks; the largest drinking quantity per time was $2.4 \pm 1.2$ drinks. The mean score \pm SD of each tool was as follows: $3.9 \pm 4.7$ in AUDIT; $0.4 \pm 0.8$ in CAGE; $0.4 \pm 0.9$ in TACE; $0.9 \pm 1.4$ in TWEAK; and $0.2 \pm 0.5$ in NET. Thirty-five persons were heavy drinkers who drank more than 7 glasses per week (8.8\%); 144 were excessive drinkers who drank more than 3 glasses per time (36.0\%), 144 were at-risk drinkers (36.00\%), and 58 were drinkers with alcohol use disorders (14.5\%) (Table 1). 
Table 1. Demographic characteristics of participants

\begin{tabular}{|c|c|}
\hline Characteristic & Value \\
\hline Age (y) & $42.2 \pm 12.8$ \\
\hline Age of alcohol contact & $22.5 \pm 6.3$ \\
\hline \multicolumn{2}{|l|}{ Religion } \\
\hline Yes & $234(58.5)$ \\
\hline No & $166(41.5)$ \\
\hline \multicolumn{2}{|l|}{ Living state } \\
\hline Housemate & $330(82.5)$ \\
\hline Alone & $70(17.5)$ \\
\hline \multicolumn{2}{|l|}{ Smoking } \\
\hline Non-smokers & $374(93.5)$ \\
\hline Ex-smokers & $19(4.8)$ \\
\hline Current-smokers & $7(1.8)$ \\
\hline \multicolumn{2}{|l|}{ Education level } \\
\hline Elementary school & $9(2.3)$ \\
\hline Middle school & $38(9.5)$ \\
\hline High school & $122(30.5)$ \\
\hline University/college & $211(52.8)$ \\
\hline >University/college & $20(5.0)$ \\
\hline Frequency of drinking per week & $0.5 \pm 0.7$ \\
\hline Usual drinking amounts on an occasion (drink) ${ }^{*}$ & $1.6 \pm 1.6$ \\
\hline Maximal drinks on an occasion (drink) & $2.4 \pm 1.2$ \\
\hline \multicolumn{2}{|l|}{ Score of screening questionnaires } \\
\hline AUDIT & $3.9 \pm 4.7$ \\
\hline AUDIT-Consumption & $2.7 \pm 2.4$ \\
\hline Cut down, Annoyed, Guilty, Eye-opener & $0.4 \pm 0.8$ \\
\hline Tolerance, Annoyed, Cut down, Eye-opener & $0.4 \pm 0.9$ \\
\hline Tolerance, Worried, Eye-opener, Amnesia, Kut down & $0.9 \pm 1.4$ \\
\hline Normal drinker, Eye-opener, Tolerance & $0.2 \pm 0.5$ \\
\hline At-risk drinking ${ }^{\dagger}$ & $144(36.0)$ \\
\hline Heavy drinking & $35(8.8)$ \\
\hline Binge drinking & $144(36.0)$ \\
\hline Alcohol use disorder & $58(14.5)$ \\
\hline
\end{tabular}

Values are presented as mean \pm standard deviation or number (\%). AUDIT, Alcohol Use Disorders Identification Test.

${ }^{*}$ One standard drink unit was considered to be $14 \mathrm{~g}$ of alcohol. ${ }^{\dagger}$ Consumption of more than 3 drinks on an occasion or 7 in 1 week.

\section{Area under Receiver Operating Characteristic of Each Question about Screening of Alcohol Use Disorder}

AUROCs of the 10 questions in AUDIT, 4 in CAGE, 5 in TWEAK, 4 in TACE, and 3 in NET were compared. Of all the questions, 10 questions with the highest AUROC (95\% confidence interval [CI]) included question no. 7 in AUDIT with 0.886 (0.850-0.915), followed by question no. 5 in AUDIT with 0.862 (0.824-0.894), questions about guilt with 0.859 (0.821-0.891), question no. 2 in AUDIT with 0.832 (0.792-0.867), question no. 3 in AUDIT with 0.829 (0.789-0.865), question no. 4 in AUDIT with 0.817 (0.741-0.893), question no. 1 in AUDIT with 0.809 (0.759-0.859), question no. 8 in AUDIT with 0.790 (0.716-0.871), question about cut-down with 0.762 (0.683-0.841), and questions about tolerance with 0.734 (0.659-0.809) (Table 2).

The existing AUDIT included a total of 7 questions. The question about guilt in CAGE, the question about cut-down in CAGE and TACE, and the question about tolerance in TWEAK and TACE had a high AUROC as well.
Table 2. AUROC of ten items with the largest AUROC in identifying alcohol use disorder

\begin{tabular}{lcc}
\hline Variable & AUROC & 95\% confidence interval \\
\hline AUDIT-7 & 0.886 & $0.850-0.915$ \\
AUDIT-5 & 0.862 & $0.824-0.894$ \\
Guilty from CAGE & 0.859 & $0.821-0.891$ \\
AUDIT-2 & 0.832 & $0.792-0.867$ \\
AUDIT-3 & 0.829 & $0.789-0.865$ \\
AUDIT-4 & 0.817 & $0.741-0.893$ \\
AUDIT-1 & 0.809 & $0.759-0.859$ \\
AUDIT-8 & 0.793 & $0.716-0.871$ \\
Cut down from CAGE, TACE & 0.762 & $0.683-0.841$ \\
Tolerance from TACE, TWEAK & 0.734 & $0.659-0.809$
\end{tabular}

AUROC, area under receiver operating characteristic; AUDIT, Alcohol Use Disorders Identification Test; CAGE, Cut down, Annoyed, Guilty, Eye-opener; TACE, Tolerance, Annoyed, Cut down, Eye-opener; TWEAK, Tolerance, Worried, Eye-opener, Amnesia, Kut down.

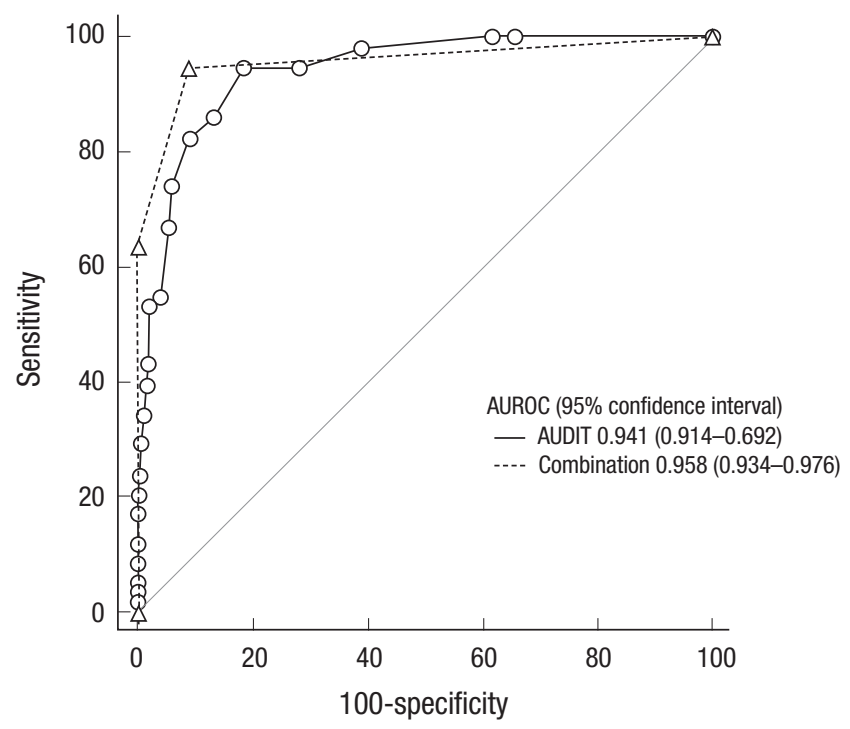

Figure 1. Comparison of receiver operating characteristic curves of the combination (AUDIT-5 and AUDIT-7) with the AUDIT in identifying alcohol use disorder. There was no significant difference in AUROC $(P=0.18)$. AUROC, area under receiver operating characteristic; AUDIT, Alcohol Use Disorders Identification Test.

\section{Comparison between AUROC of Existing Diagnostic Tools for Screening of Alcohol Use Disorders and AUROC of New Simple Combination of Questions}

AUROCs of each screening (95\% CI) were in the following order 0.941 (0.914-0.962) in AUDIT; 0.887 (0.852-0.917) in AUDIT-C; 0.883 (0.848-0.913) in CAGE; 0.871 (0.834-0.902) in TWEAK; 0.807 (0.765-0.844) in TACE; and 0.718 (0.671-0.761) in NET. AUROC of AUDIT was the broadest, with greater statistical significance than that of AUDIT-C $(\mathrm{P}<0.001)$, and there were significant differences from CAGE $(\mathrm{P}=0.014)$, TWEAK $(\mathrm{P}<0.01)$, TACE $(\mathrm{P}<0.001)$, and NET $(\mathrm{P}<0.001)$. There was no difference in AUROC of AUDIT-C as compared to those of CAGE $(\mathrm{P}=0.88)$ and TWEAK $(\mathrm{P}=0.47)$, while it was significantly greater than those of TACE $(\mathrm{P}=0.012)$ and NET $(\mathrm{P}<0.001)$. There was no difference between that of CAGE and that of TWEAK 
Table 3. Comparison of AUROC of screening questionnaires

\begin{tabular}{|c|c|c|c|c|c|c|c|}
\hline Variable & AUROC (95\% confidence interval) & AUDIT-C & CAGE & TWEAK & TACE & NET & Combination \\
\hline AUDIT & $0.941^{*}(0.914-0.969)$ & $P<0.001$ & $P=0.014$ & $P<0.01$ & $P<0.001$ & $P<0.001$ & $P=0.18$ \\
\hline AUDIT-C & $0.887(0.852-0.917)$ & & $P=0.88$ & $P=0.47$ & $P=0.012$ & $P<0.001$ & $P=0.003$ \\
\hline CAGE & $0.883(0.848-0.913)$ & & & $P=0.62$ & $P=0.002$ & $P<0.001$ & $P=0.028$ \\
\hline TWEAK & $0.871(0.834-0.902)$ & & & & $P=0.013$ & $P<0.001$ & $P=0.004$ \\
\hline TACE & $0.807(0.765-0.844)$ & & & & & $P<0.01$ & $P<0.001$ \\
\hline NET & $0.718(0.671-0.761)$ & & & & & & $P<0.001$ \\
\hline Combination & $0.958(0.934-0.976)$ & & & & & & \\
\hline
\end{tabular}

P-values were obtained by comparison of independent ROC curves. Combination was obtained by AUDIT- 5 and AUDIT-7.

AUROC, area under receiver operating characteristic; AUDIT-C, Alcohol Use Disorders Identification Test-Consumption; CAGE, Cut down, Annoyed, Guilty, Eye-opener; TWEAK, Tolerance, Worried, Eye-opener, Amnesia, Kut down; TACE, Tolerance, Annoyed, Cut down, Eye-opener; NET, Normal drinker, Eye-opener, Tolerance; AUDIT, Alcohol Use Disorders Identification Test.

$(\mathrm{P}=0.62)$, and there were significant differences from those of TACE $(\mathrm{P}=0.002)$ and NET $(\mathrm{P}<0.001)$. That of TWEAK was greater than those of TACE $(\mathrm{P}=0.013)$ and NET $(\mathrm{P}<0.001)$, and there was a significant difference between that of TACE and that of NET $(\mathrm{P}<0.001)$.

Of the 10 questions discussed above, two questions with the greatest AUROC, that is, question no. 7 in AUDIT, "Have you ever regretted after drinking?" and question no. 5 in AUDIT, "Have you ever been affected negatively by drinking in your daily life for the past year?" were chosen. AUROC of the combination of the two questions (95\% CI) was 0.958 (0.934-0.976), and there was no significant difference between that of AUDIT: 0.941 (0.914-0.962), and the greatest AUROC of the existing alcohol use disorder screening tools (Figure 1). In contrast, AUROC of the combination of the two questions was significantly broader than those of AUDIT-C $(\mathrm{P}=0.0003)$, CAGE $(\mathrm{P}=0.0028)$, TWEAK $(\mathrm{P}=0.0004)$, TACE $(\mathrm{P}<0.001)$, and NET $(\mathrm{P}<0.001)$ (Table 3$)$.

\section{DISCUSSION}

It has been reported that alcohol is more harmful to women's health than men, ${ }^{23)}$ and women often hide their drinking habits due to negative public perception, making detection and early treatment of alcohol abuse in females challenging. ${ }^{24,25)}$ Also it has been reported that women often fail to ask their primary care doctor questions about drinking due to a lack of awareness of problem drinking or the consultation hour. ${ }^{26)}$ This study created a new questionnaire through a recombination of existing questionnaires and the combination showed significance equal to the existing screening tools.

Among the existing screening tools, AUDIT was found to have the greatest AUROC 0.941 (0.914-0.962). And AUROC of the our newly drawn simple combination of questions no. 7 and no. 5 in AUDIT was 0.958 (0.934-0.976), noted that it would be a tool for problem drinking screening comparable to AUDIT. Previous studies to screen problem drinking in a short period of time at outpatients clinic have been performed, and new attempts have been continuously made to screen for drinking problems with one or two questions. Studies have been conducted using various methods for screening, including: using only question no. 3 of the AUDIT about the frequency of binge drinking; ${ }^{20,27,28)}$ the QF method, ${ }^{14)}$ which assesses the status of at-risk drinking based on alcohol intake calculated through drinking quantity and frequency; a method regarding the last time of binge drinking ${ }^{15)}$ that screens problem drinking by asking the questions, "When was the last time you drank more than X units of alcohol?" (in general, 5 units for men and 4 units for women) and, "When was the last time that you drank that much within 3 months"; and a method asking the question, ${ }^{29)}$ "What was the largest quantity you drank within the past 3 months?" There was an attempt to screen problem drinking only using question no. 3 in AUDIT for men in a study carried out in the US, and AUROCs of the entire questions and question no. 3 in AUDIT were respectively 0.81 and $0.795 .{ }^{20)}$ In a study on women, ${ }^{27)}$ AUROCs of AUDIT and Question no. 3 only on the screening of alcohol use disorders were respectively 0.80 and 0.76 . In a study on visitors to the emergenct room, questions about alcohol intake (quantity-frequency method) calculated with the last time, quantity and frequency of excessive drinking, AUROCs on alcohol use disorders were respectively 0.70 and 0.76 , which suggests that the QF method might be useful for primary screening. ${ }^{14)}$ In Korea, Nam et al. ${ }^{29)}$ published a problem drinking screening tool with a single question about binge drinking. AUROCs of the largest quantity within 3 months ( 4 units for men and 3 units for women based on the standard drink unit), the frequency of binge drinking within 3 months, and the number of times of intoxication per week were respectively $0.937,0.906$, and 0.768 in men and 0.970 , 0.915 , and 0.764 in women. In addition, it was noted that the question about the largest quantity within 3 months accounted for the largest area in both sexes regarding alcohol use disorders. These results support the assertion that reinforced problem drinking screening tools using simple questions are as good as the existing tools.

The details of the two questions asked were questions about "negative impacts on daily life" and "regret about drinking," unlike the quantity of heavy drinking or excessive drinking to 
which the existing simple questions attached importance. It is judged that this has been caused by changes in both women's gender roles and the criteria for diagnosing alcohol use disorders in South Korea. Most of the existing studies of problem drinking have been conducted on men. However, it is known that there are differences in psycho-social characteristics of drinking behaviors between men and women. Women tend to drink alone secretly and say that they experience shame, guilt, and decreased self-esteem due to repeated drinking. Korean society is still strongly influenced by a Confucian value system based on patriarchy and strict gender roles. ${ }^{30)}$ Traditionally, in fact, Confucianism forbids alcohol consumption by anyone except adult males. Of the various questions, question no. 5 in AUDIT, "How often during the last year have you failed to do what was normally expected from you because of drinking?" and question no. 7 in AUDIT, "How often during the last year have you had a feeling of guilt or remorse after drinking?" were included in light of the fact that a negative perception of alcohol consumption by women still exists in Korean society, even though it has become permitted due to recent social and cultural changes. Also each question seems to be related to the items, "Important social, occupational, or recreational activities are given up or reduced due to drinking" and "Repeated drinking prevents one from performing important tasks at the workplace, school, or home" among the criteria for the diagnosis of alcohol use disorders. This seems to be related to the designation of more than two symptoms as alcohol use disorder according to the DSM-5.

In addition, regarding the results of a review of the usefulness of the existing tools in this study, it was found that AUROC of AUDIT had significant differences from those of AUDIT-C, CAGE, TWEAK, TACE, and NET. There was no significant difference between CAGE and TWEAK, while TACE showed significant difference in comparison to CAGE and TWEAK. This seems to be the result of the same eye-opener question in addition to the guilty, cut down, and tolerance questions that show a relatively high AUROC. The difference in each screening tool seems to be the result of the guilty question with a relatively high AUROC.

Globally, several comparative studies have been carried out concerning the usefulness of the existing screening. AUDIT-C, known as a typical simple screening tool, is reported to have a lower capacity for screening alcohol use disorders as compared to AUDIT. According to a study carried out on the usefulness of AUDIT-C in 1998, ${ }^{18)}$ AUROCs of AUDIT and AUDIT-C were respectively 0.81 and 0.79 , which showed that the capacity of screening problem drinking by AUDIT-C was significantly lower than that of AUDIT. Also in a study carried out in the UK, ${ }^{31}$ ) the capacity of screening problem drinking by AUDIT-C was significantly lower than that of AUDIT for women; and CAGE, too, had a lower capacity for screening problem drinking than that of AUDIT. According to this study, AUROCs of CAGE, AUDIT-C, and AUDIT were respectively 0.76, 0.82, and 0.87; and it was found that, among the screening tools, AUDIT had a high diagnostic value. In a study carried out in the US, ${ }^{32)}$ AUROC of CAGE for women was 0.77 , showing a significant difference from 0.94, that of AUDIT. However, there was a study showing that AUDIT-C was a comparable screening tool to AUDIT. In a study of American women, AUROCs of AUDIT and AUDIT-C on alcohol use disorders were respectively 0.90 and 0.91 , which demonstrates that the capacity of screening problem drinking by 10 -question AUDIT or AUDIT-C are almost equal. ${ }^{25)}$ Another study showed no difference between AUROCs of AUDIT and AUDIT-C in detecting women's alcohol use disorders, which were respectively 0.94 and $0.91 .^{32)}$ Based on the above findings, AUDIT would be the most appropriate as a female problem drinker screening tool among the existing tools.

It was found in this study that the ratio of at-risk drinking was $36.0 \%$, and the ratio of alcohol use disorders according to DSM5 diagnostic criteria was $14.5 \%$. It has been reported that the ratio of female alcohol use disorders is $19.5 \%$ in the US ${ }^{33)}$ and $6.1 \%$ in South Korea. ${ }^{34)}$

The limitations of this study are as follows: First, the subjects of the study were women who visited a single hospital at a certain period of time, making it difficult to apply the results of this study universally to the female population. Secondly, memory bias may have affected the survey results, particularly in the items of alcohol intake and frequency. Finally, the reliability for the tools' examination and re-examination method was not verified, indicating a need for further research in the future.

In spite of these limitations, in reality, there is currently no simple alcohol use disorder screening tool for the female population. The results of this study are significant in that they imply that a simple combination of two questions can be used for screening women's alcohol use disorders in the primary care setting.

\section{CONFLICT OF INTEREST}

No potential conflict of interest relevant to this article was reported.

\section{REFERENCES}

1. Lee DH. Korea alcohol consumption habits 2012 [Internet]. Cheongju: Ministry of Food and Drug Safety Food and Drug Administration; 2012 [cited 2014 May 20]. Available form: http://www.mfds.go.kr/index.do?x $=0 \&$ searchkey=title: contents \&mid $=675 \&$ searchword $=\% \mathrm{C} 1 \% \mathrm{D} 6 \% \mathrm{~B} 7 \%$ F9\&y $=0 \&$ pageNo $=2 \&$ seq $=19264 \& \mathrm{cmd}=\mathrm{v}$.

2. Ministry of Health and Welfare. Korea health statistics 2012: Korea National Health and Nutrition Examination Survey (KNHANES V-3) [Internet]. Cheongju: Korea Centers for Disease Control and Prevention; 2012 [cited 2014 May 20]. Available form: https://knhanes.cdc.go.kr/ 
knhanes/sub04/sub04_03.do?classType=7.

3. Kahan M, Wilson L, Becker L. Effectiveness of physician-based interventions with problem drinkers: a review. CMAJ 1995;152:851-9.

4. Fleming MF, Barry KL, Manwell LB, Johnson K, London R. Brief physician advice for problem alcohol drinkers: a randomized controlled trial in community-based primary care practices. JAMA 1997;277:103945.

5. Roche AM, Richard GP. Doctors' willingness to intervene in patients' drug and alcohol problems. Soc Sci Med 1991;33:1053-61.

6. Saunders JB, Aasland OG, Babor TF, de la Fuente JR, Grant M. Development of the Alcohol Use Disorders Identification Test (AUDIT): WHO Collaborative Project on Early Detection of Persons with Harmful Alcohol Consumption--II. Addiction 1993;88:791-804.

7. Mayfield D, McLeod G, Hall P. The CAGE questionnaire: validation of a new alcoholism screening instrument. Am J Psychiatry 1974;131:1121-3.

8. Sokol RJ, Martier SS, Ager JW. The T-ACE questions: practical prenatal detection of risk-drinking. Am J Obstet Gynecol 1989;160:863-8.

9. Sarkar M, Einarson T, Koren G. Comparing the effectiveness of TWEAK and T-ACE in determining problem drinkers in pregnancy. Alcohol Alcohol 2010;45:356-60.

10. Chang G. Alcohol-screening instruments for pregnant women. Alcohol Res Health 2001;25:204-9.

11. Russell M, Martier SS, Sokol RJ, Mudar P, Bottoms S, Jacobson S, et al. Screening for pregnancy risk-drinking. Alcohol Clin Exp Res 1994;18: 1156-61.

12. Fiellin DA, Reid MC, O'Connor PG. Screening for alcohol problems in primary care: a systematic review. Arch Intern Med 2000;160:1977-89.

13. Bohn MJ, Babor TF, Kranzler HR. The Alcohol Use Disorders Identification Test (AUDIT): validation of a screening instrument for use in medical settings. J Stud Alcohol 1995;56:423-32.

14. Canagasaby A, Vinson DC. Screening for hazardous or harmful drinking using one or two quantity-frequency questions. Alcohol Alcohol 2005;40:208-13.

15. Williams R, Vinson DC. Validation of a single screening question for problem drinking. J Fam Pract 2001;50:307-12.

16. National Institute on Alcohol Abuse and Alcoholism. Helping patients who drink too much: a clinician's guide [Internet]. Bethesda (MD): National Institutes of Health Publication; 2007 [cited 2014 May 20]. Available from: http://pubs.niaaa.nih.gov/publications/Practitioner/ Clinic iansGuide2005/guide.pdf.

17. American Psychiatric Association. Diagnostic and statistical manual of mental disorders. 5th ed. Washington (DC): American Psychiatric Press; 2013.

18. Kim JS, Kim SS, Jung JK, Yoon SJ. Essentials of family medicine for medical student. Daejeon: Chungnam National University Publishers; 2010.

19. Kim CG, Kim JS, Jung JG, Kim SS, Yoon SJ, Suh HS. Reliability and validity of Alcohol Use Disorder Identification Test-Korean revised version for screening at-risk drinking and alcohol use disorders. Korean J Fam Med 2014;35:2-10.

20. Bush K, Kivlahan DR, McDonell MB, Fihn SD, Bradley KA. The AUDIT alcohol consumption questions (AUDIT-C): an effective brief screening test for problem drinking. Ambulatory Care Quality Improvement Project (ACQUIP). Alcohol Use Disorders Identification Test. Arch Intern Med 1998;158:1789-95.

21. Dawson DA, Grant BF, Stinson FS, Zhou Y. Effectiveness of the derived Alcohol Use Disorders Identification Test (AUDIT-C) in screening for alcohol use disorders and risk drinking in the US general population. Alcohol Clin Exp Res 2005;29:844-54.

22. Bottoms SF, Martier SS, Sokol RJ. Refinements in screening for risk drinking in reproductive-aged women: the "NET" results. Alcohol Clin Exp Res 1989;13:339.

23. Lee E, Park SJ. Women's alcohol dependence: clinical characteristics and treatment. J Korean Acad Addict Psychiatry 2006;10:86-90.

24. Lim SY, Cho HS, Lee YH. A case study about female alcoholic's alcohol addictive process. Korean J Clin Psychol 2005;24:869-86.

25. Timko C, Finney JW, Moos RH. The 8-year course of alcohol abuse: gender differences in social context and coping. Alcohol Clin Exp Res 2005;29:612-21.

26. Wallace P, Cutler S, Haines A. Randomised controlled trial of general practitioner intervention in patients with excessive alcohol consumption. BMJ 1988;297:663-8.

27. Bradley KA, Bush KR, Epler AJ, Dobie DJ, Davis TM, Sporleder JL, et al. Two brief alcohol-screening tests From the Alcohol Use Disorders Identification Test (AUDIT): validation in a female Veterans Affairs patient population. Arch Intern Med 2003;163:821-9.

28. Smith PC, Schmidt SM, Allensworth-Davies D, Saitz R. Primary care validation of a single-question alcohol screening test. J Gen Intern Med 2009;24:783-8.

29. Nam TW, Kim JS, Kim SS, Jung JG, Kang DS, Hyeon YH, et al. Utility of single alcohol questions related to binge drinking in identifying problem drinkers. Korean J Fam Med 2009;30:777-83.

30. Park HR, Lee JJ. Research on culture of women's alcohol consumption: when women drink? Korean J Women Psychol 2004;9;39-52.

31. Aertgeerts B, Buntinx F, Ansoms S, Fevery J. Screening properties of questionnaires and laboratory tests for the detection of alcohol abuse or dependence in a general practice population. Br J Gen Pract 2001; 51:206-17.

32. Bradley KA, DeBenedetti AF, Volk RJ, Williams EC, Frank D, Kivlahan DR. AUDIT-C as a brief screen for alcohol misuse in primary care. Alcohol Clin Exp Res 2007;31:1208-17.

33. Hasin DS, Stinson FS, Ogburn E, Grant BF. Prevalence, correlates, disability, and comorbidity of DSM-IV alcohol abuse and dependence in the United States: results from the National Epidemiologic Survey on Alcohol and Related Conditions. Arch Gen Psychiatry 2007;64:830-42.

34. Ministry of Health and Welfare. The 2011 epidemiological survey of mental disorders among Korean adults [Internet]. Sejong: Ministry of Health and Welfare; 2011 [cited 2014 May 20]. Available form: http:// www.bokjiro.go.kr/cmm/fms/FileDown.do?atchFileId=5769251\&file $\mathrm{Sn}=6986.9 \% 20 \mathrm{~KB}$. 